\title{
MEROMORPHIC FUNCTIONS WITH ADDITION OR MULTIPLICATION THEOREMS*
}

\author{
BY
}

J. F. RITT

It is a theorem of Briot and Bouquet that if a solution of an algebraic differential equation of the first order,

$$
F\left(\frac{d w}{d z}, w\right)=0
$$

is uniform, the solution is a rational function, or a rational function of $e^{\mu z}$ ( $\mu$ constant), or an elliptic function. It is part of a famous theorem of Weierstrass that every uniform function with an algebraic addition theorem is of one of the three types just enumerated. It is a result of Picard that if two meromorphic functions are algebraically related to one another, either the relation is of genus zero, in which case the two functions are rational functions of a single meromorphic function, or the relation is of genus unity, in which case the two functions are elliptic functions of a single integral function. $\dagger$

The present paper treats a problem which might easily be suggested by any of the above theorems. We seek all meromorphic functions $f(z)$ such that, for some linear $\lambda(z)$, an algebraic relation

$$
F(f[\lambda(z)], f(z))=0
$$

holds.

There is no generality lost in assuming $\lambda(z)$ to be of one of the forms $z+m$ or $m z$. Thus our problem is related to difference and $q$-difference equations as that of Briot and Bouquet is to differential equations. Of course, the assumption made here that $f(z)$ is meromorphic is stronger than the requirement of mere uniformity in the differential equation problem. For the case in which $\lambda(z)=z+m$, we have, as we are dealing with a single value

* Presented to the Society, February 27, 1926; received by the editors in March, 1926.

† Acta Mathematica, vol. 11 (1887), p. 1. Picard's theorem deals with any pair of uniform functions with an isolated essential singularity. See also Bulletin des Sciences Mathématiques, vol. 15 (1880), p. 416, where an incomplete proof of the theorem is given for meromorphic functions. The proof in that paper that when the relation is of genus unity the functions are elliptic functions of an integral function, is rigorous. 
of $m$, a generalization of Weierstrass's addition theorem problem, to be sure with a heavy function-theoretic restriction. Of course, our investigation also amounts to the further study of the two functions in Picard's theorem when one of the functions is found from the other by transforming the variable linearly.

In stating our results, we assume that $\lambda(z)=m z$ with $m$ a constant not 0 or 1 , or that $\lambda(z)=z+m$ with $m$ a constant not 0 . As has already been said, this involves no sacrifice of generality.

The relation between $f(z)$ and $f[\lambda(z)]$ is, by Picard's theorem, either of genus unity or of genus zero. For the case in which $f(z)$ and $f[\lambda(z)]$ satisfy a relation of genus unity, we prove that $f(z)$ is an elliptic function of an integral function $g(z)$, where $g[\lambda(z)]$ is a linear function of $g(z)$. Explicit expressions are given for $g(z)$ in $\$ 4$.

The case of genus zero is more complicated. There are the following possibilities:

(a) $f(z)$ is an elliptic function of an integral function $g(z)$, with $g[\lambda(z)] a$ linear function of $g(z)$.

(b) $f(z)$ is a rational function of a meromorphic function $h(z)$, where some one of the functions $h(z), h[\lambda(z)]$ is a rational function of the other.

If $\lambda(z)=m z, h(z)$ belongs to a class of functions discussed by Poincaré in a paper on rational multiplication theorems. ${ }^{*}$ Poincaré's work tells enough about these functions for them to be regarded as known. If $\lambda(z)=z+m$, $h(z)$ is a function with a rational addition theorem. No complete determination of such functions has thus far been made. A large class of them can be obtained from Poincarés functions by a substitution, but it is by no means certain that they are all found in this manner.

(c) $f(z)$ is a rational function of $h\left[(g(z))^{1 / n}\right]$, where $n$ is an integer, $g(z)$ an integral function such that $(g[\lambda(z)])^{1 / n}$ is linear in $(g(z))^{1 / n}$, and where $h(z)$ is one of the following periodic meromorphic functions: $e^{z}, \cos z, \varphi(z)$; in the lemniscatic case $\left(g_{3}=0\right), g^{2}(z+u)$; in the equianharmonic case $\left(g_{2}=0\right)$, $\varphi^{\prime}(z)$ and $\varphi^{3}(z+u)$.

There are in each case a finite number of possibilities for $n$ and $u$, which are stated in detail in II. The possibilities for $g(z)$ are also determined. Both $h\left[(g(z))^{1 / n}\right]$ and $h\left[(g[\lambda(z)])^{1 / n}\right]$ will be rational in a third function $h\left[p(g(z))^{1 / n}\right.$

* Journal de Mathématiques, (3), vol. 55 (1890). 
$+q$ ], but neither of the first two functions will be a rational function of the other.*

Case (c) provides most of the work in this paper. It turns out that our problem is in a close relation with the problem of determining all pairs of rational functions $\Phi(z)$ and $\Psi(z)$ such that $\Phi[\Psi(z)]=\Psi[\Phi(z)]$, a question which the writer has studied in an earlier paper. $\dagger$ Two certain sequences of rational functions which occur in the permutable function problem appear again in this paper. Direct application is made here of the information previously obtained in regard to those sequences.

A question which we do not settle is whether Case (a) gives any functions $f(z)$ not given by Cases (b) and (c). This depends on whether certain algebraic relations derived from the relation between $f(z)$ and $f[\lambda(z)]$ are of genus unity rather than of genus zero. $\ddagger$ Except for this point, and for the question raised under Case (b) as to functions with rational addition theorems, our investigation is complete.

\section{RELATIONS OF GENUS UNITY}

1. Discussion of $\lambda(z)$. Let $f(z)$ be a non-rational meromorphic function and $\lambda(z)$ a non-constant and non-identical linear function. If $f(z)$ and $f[\lambda(z)]$ satisfy an algebraic relation, then $\lambda(z)$ must be integral, that is $\lambda(z)=m z+n$ with $m$ and $n$ constant. For if $\lambda(z)$ had a pole at a finite point $\alpha, f[\lambda(z)]$, and therefore also $f(z)$, would have an essential singularity at $\alpha$.

If $m \neq 1$, we write

$$
f_{1}(z)=f\left(z+\frac{n}{1-m}\right) .
$$

Then $f_{1}(z)$ and $f_{1}(m z)$ obey the same relation as do $f(z)$ and $f[\lambda(z)]$. TWe therefore assume that $\lambda(z)$ is of one of the forms $m z$ or $z+m$, throwing away no generality in the assumption.

2. Relations of genus unity. We assume in this section that $f(z)$ and $f[\lambda(z)]$ satisfy a relation of genus one.

Let $\lambda_{2}(z)=\lambda[\lambda(z)]$. Evidently $f\left[\lambda_{2}(z)\right]$ is algebraic in $f(z)$.

* For instance, the relation between $\cos (4 z)^{1 / 2}$ and $\cos z^{1 / 2}$ comes under Case (b), whereas that between $e^{\text {ss/2 }}$ and $e^{z}$ comes under Case (c).

$\dagger$ Ritt, Permutable rational functions, these Transactions, vol. 25 (1923), p. 399.

$\ddagger$ See $\$ 6$. 
As $f[\lambda(z)]$ and $f\left[\lambda_{2}(z)\right]$ are both algebraic in $f(z)$, we can find a rational combination of $f[\lambda(z)]$ and $f\left[\lambda_{2}(z)\right]$-call it $h(z)$-such that $f[\lambda(z)]$ and $f\left[\lambda_{2}(z)\right]$ are both rational combinations of $f(z)$ and $h(z){ }^{*}$

Then $f(z)$ and $h(z)$ are meromorphic functions between which there exists an algebraic relation. This relation cannot be of genus zero. If it were, $f(z)$ and $h(z)$ would both be rational functions of a single meromorphic function. Consequently $f[\lambda(z)]$, rational in $f(z)$ and $h(z)$, would also be rational in this new meromorphic function, so that the relation between $f(z)$ and $f[\lambda(z)]$ would have to be of genus zero.

Hence $f(z)$ and $h(z)$ satisfy a relation of genus unity, and are elliptic functions of an integral function $g(z)$. Then $f[\lambda(z)]$ and $f\left[\lambda_{2}(z)\right]$ are also elliptic functions of $g(z)$. Let

$$
f(z)=\phi_{1}[g(z)], \quad f[\lambda(z)]=\phi_{2}[g(z)], \quad f\left[\lambda_{2}(z)\right]=\phi_{3}[g(z)],
$$

where $\phi_{1}(z), \phi_{2}(z)$, and $\phi_{3}(z)$ are elliptic functions.

Let the relation of genus unity which connects $f(z)$ and $f[\lambda(z)]$ be

$$
F(u, v)=0,
$$

that is, let (2) be satisfied when $u$ and $v$ are replaced by $f(z)$ and $f[\lambda(z)]$ respectively. Then (2) is satisfied when $u$ is replaced by $\phi_{1}(z)$ and $v$ by $\phi_{2}(z)$. Since $f[\lambda(z)]$ and $f\left[\lambda_{2}(z)\right]$ obey (2), we can also satisfy (2) by replacing $u$ and $v$ by $\phi_{2}(z)$ and $\phi_{3}(z)$ respectively.

Let

$$
\int R(u, v) d u
$$

be an integral of the first kind attached to the relation (2). If, in the expression

$$
R(u, v) \frac{d u}{d z}
$$

we replace $u$ and $v$ by $\phi_{1}(z)$ and $\phi_{2}(z)$ respectively, or by $\phi_{2}(z)$ and $\phi_{3}(z)$ respectively, we obtain an elliptic function whose integral with respect to $z$ could not become infinite for any finite $z$. $\dagger$ The elliptic function thus obtained must be a constant.

* Weber, Algebra, 2d edition, vol. 1, p. 499.

† Cf. Picard, Traité d'A nalyse, 2d edition, vol. 2, p. 561. 
We shall replace $u$ and $v$ in (2) by $f[\lambda(z)]$ and $f\left[\lambda_{2}(z)\right]$ respectively. Using (1), we see that the result is a constant times the derivative of $g(z)$. But if we use the expressions

$$
f[\lambda(z)]=\phi_{1}(g[\lambda(z)]), \quad f\left[\lambda_{2}(z)\right]=\phi_{2}(g[\lambda(z)]),
$$

we find the same result expressed as a constant times the derivative of $g[\lambda(z)]$.

Hence

$$
\frac{d}{d z} g[\lambda(z)]=p \frac{d}{d z} g(z)
$$

with $p$ a constant, so that

$$
g[\lambda(z)]=p g(z)+q,
$$

with $q$ constant, as well as $p$. We have thus, for the case of genus unity, the result stated in the introduction.

3. The constants $p$ and $q$. Equation (3) leads quickly to explicit expressions for $g(z)$. Before giving these expressions, let us examine the constants $p$ and $q$, assuming $\phi_{1}(z)$ to have been given as any elliptic function.

We shall fix $p$ and $q$ so that $\phi_{1}(z)$ and $\phi_{1}(p z+q)$ may be algebraically related, without insisting that the relation between them be of genus unity. It is necessary and sufficient that $\phi_{1}(z)$ and $\phi_{1}(p z+q)$ have a pair of periods in common. There is no restriction on $q$. Any rational value of $p$ is admissible, and when the ratio of the periods of $\phi_{1}(z)$ is a quadratic number, there is a system of complex values for $p$.

If, $\lambda(z)$ being given, we use every elliptic function $\phi_{1}(z)$, every $p$ and $q$ described as above, and every integral $g(z)$ satisfying (3), we shall obtain every meromorphic $f(z)$ which satisfies with $f[\lambda(z)]$ a relation of genus unity. But for certain choices of $\phi_{1}(z), p$ and $q$, the relation will be of genus zero.*

4. The function $g(z)$. We shall now discuss $g(z)$ and $\lambda(z)$, regarding $p$ and $q$ as known. The results will be used again in II.

Suppose first that $\lambda(z)=m z$. Let $g(z)$ have, for $z=0$, the development

$$
g(z)=a+b z^{n}+c z^{n+1}+\cdots,
$$

where $b \neq 0$.

We must have $m^{n}=p$. Suppose first that $p$ (and therefore $m$ ) is not a rational root of unity. All terms after the second in $g(z)$ disappear, and we have

\footnotetext{
* For instance, let $\phi_{1}(z)$ be $\varphi(z), p$ be rational and $q$ be zero.
} 


$$
g(z)=\frac{q}{1-p}+b z^{n},
$$

with $b$ and $n$ arbitrary.

Let $p$ be a root of unity. Taking any positive integral $n$, we may choose for $m$ any $n$th root of $p$. Let $m$ thus chosen be a primitive $i$ th root of unity. We must have

$$
g(z)=a+z^{n} h\left(z^{i}\right),
$$

with $h(z)$ an integral function. We cannot have $p=1$, unless $q=0$. When $p \neq 1$, we have $a=q /(1-p)$, whereas $n$ and $h(z)$ are arbitrary. If $p=1$, $q=0, a$ becomes arbitrary.

Suppose now that $\lambda(z)=z+m$. When $p \neq 1$, we find, by elementary considerations, that

$$
g(z)=e^{z(\log p) / m} \pi(z)-\frac{q}{p-1},
$$

where $\pi(z)$ is any periodic integral function with $m$ for period. Any value distinct from zero can be used for $m$. When $p=1$, we find that

$$
g(z)=\frac{q}{m} z+\pi(z)
$$

with $\pi(z)$ and $m$ as above.

\section{Relations OF GENUS zeRo}

5. The functions $f_{i}(z)$. If $\phi(z)=\phi_{1}\left[\phi_{2}(z)\right]$, where $\phi_{1}(z)$ and $\phi_{2}(z)$ are rational and $\phi_{2}(z)$ is not linear, we shall call $\phi_{2}(z)$ a forefactor of $\phi(z)$.

Let the meromorphic functions $f_{0}(z)$ and $f_{0}[\lambda(z)], \lambda$ linear, satisfy a relation of genus zero. We have

$$
f_{0}(z)=\phi_{0}\left[f_{1}(z)\right], \quad f_{0}[\lambda(z)]=\psi_{0}\left[f_{1}(z)\right],
$$

with $f_{1}(z)$ meromorphic and $\phi_{0}(z)$ and $\psi_{0}(z)$ rational.

It is possible so to choose $f_{1}(z)$ that $f_{1}(z)$ is a rational function of $f_{0}(z)$ and $f_{0}[\lambda(z)]$.* We suppose this to be done. Then $\phi_{0}(z)$ and $\psi_{0}(z)$ have no forefactor in common. For if they had a common forefactor $\sigma(z)$, then $f_{1}(z)$, as a rational combination of $f_{0}(z)$ and $f_{0}[\lambda(z)]$, would be a rational function of $\sigma\left[f_{1}(z)\right]$.

As

$$
f_{0}[\lambda(z)]=\psi_{0}\left[f_{1}(z)\right]=\phi_{0}\left(f_{1}[\lambda(z)]\right),
$$

* Appell et Goursat, Theorie des Fonctions Algébriques, Paris, 1895, p. 288. 
$f_{1}(z)$ and $f_{1}[\lambda(z)]$ are algebraically related. The relation which they satisfy is of genus unity or of genus zero. If it is of genus unity, $f_{1}(z)$ is an elliptic function of an integral function $g(z)$, where $g(z)$ is described as in $\$ 4$. Of course, $f_{0}(z)$ will also be an elliptic function of $g(z)$.

Suppose that $f_{1}(z)$ and $f_{1}[\lambda(z)]$ satisfy a relation of genus zero. Then there exist a meromorphic function $f_{2}(z)$, and two rational functions, $\phi_{1}(z)$ and $\psi_{1}(z)$, with no forefactor in common, such that

$$
f_{1}(z)=\phi_{1}\left[f_{2}(z)\right], \quad f_{1}[\lambda(z)]=\psi_{1}\left[f_{2}(z)\right] .
$$

6. Case (a). We continue the above procedure. It is conceivable that, for certain functions $f_{0}(z)$, we reach at some step an $f_{i}(z)$ which satisfies with $f_{i}[\lambda(z)]$ a relation of genus unity. If such functions $f_{0}(z)$ exist, they are elliptic functions of integral functions.

Whether a relation of genus unity is ever reached for certain functions, is a question which we do not settle in this paper. But certainly there exist functions $f_{0}(z)$ which are elliptic functions of integral functions and which satisfy with $f_{0}[\lambda(z)]$, for some non-identical $\lambda(z)$, a relation of genus zero. This makes it legitimate to create Case (a) under a genus zero, in our statement of results in the introduction. With Case (a) established, our results assume a simple form. But if it should be that no relation of genus zero leads to a relation of genus unity, then Case (a) may be suppressed.

7. The sequences (I) and (II). We shall assume in what follows that at no step are we led to a relation of genus unity. Then there exist two infinite sequences of rational functions

$$
\begin{array}{llll}
\phi_{0}(z), & \phi_{1}(z), & \phi_{2}(z), \cdots, & \phi_{i}(z), \cdots, \\
\psi_{0}(z), & \psi_{1}(z), & \psi_{2}(z), \cdots, & \psi_{i}(z), \cdots,
\end{array}
$$

where, for every $i, \phi_{i}(z)$ and $\psi_{i}(z)$ have no common forefactor, and an infinite sequence of meromorphic functions

$$
f_{0}(z), \quad f_{1}(z), \quad f_{2}(z), \cdots, \quad f_{i}(z), \cdots,
$$

such that, for every $i$,

$$
f_{i}(z)=\phi_{i}\left[f_{i+1}(z)\right], \quad f_{i}[\lambda(z)]=\psi_{i}\left[f_{i+1}(z)\right] .
$$

As

$$
\begin{aligned}
& f_{i}[\lambda(z)]=\phi_{i}\left(f_{i+1}[\lambda(z)]\right)=\phi_{i}\left(\psi_{i+1}\left[f_{i+2}(z)\right]\right), \\
& f_{i}[\lambda(z)]=\psi_{i}\left[f_{i+1}(z)\right]=\psi_{i}\left(\phi_{i+1}\left[f_{i+2}(z)\right]\right),
\end{aligned}
$$

we have, for every $i$,

$$
\phi_{i}\left[\psi_{i+1}(z)\right]=\psi_{i}\left[\phi_{i+1}(z)\right]
$$


8. The degrees $r$ and $s$. At this point we shall begin to use the methods and results of our paper Permutable rational functions, to which reference has already been made. This paper will be called $A$.

We represent the degrees of $\phi_{i}(z)$ and $\psi_{i}(z)$ by $r_{i}$ and $s_{i}$ respectively. We shall prove that, for every $i$,

$$
r_{i+1} \leqq r_{i}, \quad s_{i+1} \leqq s_{i} .
$$

We write

$$
w=G(z)=\phi_{i}\left[\psi_{i+1}(z)\right]=\psi_{i}\left[\phi_{i+1}(z)\right] .
$$

The function $\psi_{i+1}(z)$ determines $r_{i}$ systems of imprimitivity of the group of monodromy of $G^{-1}(w)$, each consisting of $s_{i+1}$ letters. ${ }^{*}$ Similarly $\phi_{i+1}(z)$ determines $s_{i}$ such systems, each system consisting of $r_{i+1}$ letters. If we had $\boldsymbol{r}_{i+1}>\boldsymbol{r}_{i}$, some system determined by $\phi_{i+1}(z)$ would have more than one letter in common with at least one of the systems determined by $\psi_{i+1}(z)$. This would mean that $\phi_{i+1}(z)$ and $\psi_{i+1}(z)$ have a common forefactor. $\dagger$ Hence $r_{i+1} \leqq r_{i}, s_{i+1} \leqq s_{i}$.

9. Paper $A$. There occur in $A$, two sequences of rational functions, called $(A)$ and $(B)$,

$$
\phi_{0}, \phi_{1}, \phi_{2}, \cdots, \quad(B) \quad \psi_{0}, \psi_{1}, \psi_{2}, \cdots,
$$

whose theory is practically identical with that of the sequences (I) and (II) of this paper. In studying the sequences $(A)$ and $(B)$, we made use of the following facts:

(a) The degrees of the functions in both sequences are non-increasing.

(b) For every $i, \phi_{i}$ and $\psi_{i}$ have no common forefactor.

(c) For every $i, \phi_{i} \psi_{i+1}=\psi_{i} \phi_{i+1} . \neq$

From (a) it followed immediately that, for sufficiently large values of $i$, the degrees of $\phi_{i}$ and $\psi_{i}$ do not change with $i$. We proved also, using (b) and (c), that there exist two integers, $h \leqq 4$ and $k \leqq 4$, such that, for $i$ sufficiently large, the inverse of $\phi_{i}$ has precisely $h$ critical points and that of $\psi_{i}$ precisely $k$ critical points. We then determined, with the help of (b) and (c), the nature of the critical points of the inverses $\phi_{i}^{-1}$ and $\psi_{i}^{-1}$.

As the sequences (I) and (II) have the properties (a), (b) and (c), the discussion of the critical points of $\phi_{i}^{-1}$ and $\psi_{i}^{-1}$ in $(A)$ and $(B)$ can be

\footnotetext{
* $A$, p. 403. If $\psi_{i+1}$ is linear, we have systems of one letter. Of course, such systems are not commonly called systems of imprimitivity.

$\dagger A$, p. 405, line 26.

$\ddagger$ Of course, (c) and (b) imply (a). But (a) was not derived from (b) and (c) in paper $A$, so that the proof of (a) in $\$ 8$ is not a redundancy.
} 
carried over without modification to the sequences (I) and (II). We are in a position to state, for $i$ sufficiently large, how the branches of $\phi_{i}^{-1}$ and $\psi_{i}^{-1}$ in (I) and (II) are permuted at their critical points.

In what follows, we shall consider in turn the several possibilities for $\phi_{i}$ and $\psi_{i}$, consulting $A$ at each step. But we must first stop to consider the case in which, for $i$ sufficiently large, the functions in one of the sequences (I) and (II) are linear.

10. Case (b). Suppose that, for some $i$, one or both of the functions $\phi_{i}, \psi_{i}$ are linear. There is no generality lost in assuming, when $\phi_{i}$ and $\psi_{i}$ are not both linear, that $\phi_{i}$ is linear rather than $\psi_{i}$. If necessary, we can study the pair of functions $f(z)$ and $f\left[\lambda^{-1}(z)\right]$, thus interchanging the rôles of (I) and (II). We suppose then that $\phi_{i}$ is linear.

As we have a linear function at our disposal in choosing $f_{i+1}(z)$, we may evidently suppose that $\phi_{i}(z)=z$, so that $f_{i+1}(z)=f_{i}(z)$, and

$$
f_{i}[\lambda(z)]=\psi_{i}\left[f_{i}(z)\right] .
$$

In the case of $\lambda(z)=m z, f_{i}(z)$ is a meromorphic function with a rational multiplication theorem. Such functions are so thoroughly described by an existence theorem of Poincaré, ${ }^{*}$ that they may be regarded as completely determined.

In stating this theorem, we shall assume that $f_{i}(0)=0$. This can always be brought about by replacing $f_{i}(z)$ by a linear function of itself. It is easy to prove that, in a relation like (4), $\psi_{i}^{\prime}(0)$ must be an integral power of $m$. Poincaré shows that, given any rational $\psi_{i}(z)$ with $\psi_{i}(0)=0$ and $\left|\psi_{i}^{\prime}(0)\right|>1$, and given an $m$ such that $m^{n}=\psi_{i}^{\prime}(0)(n$ a positive integer), there exists a meromorphic $f_{i}(z)$ with $n$ zeros at the origin which satisfies (4). Every other solution of (4) is obtained from this particular solution by replacing $z$ by a constant times $z$.

In a relation (4) with $|m| \leqq 1, \psi_{i}(z)$ must be linear. Relinquishing the condition that $f_{i}(0)=0$, we may, replacing $f_{i}(z)$ by a suitable linear function of itself, so arrange matters that $\psi_{i}(z)$ is of the form $p z+q$. The discussion of $f_{i}(z)$ then becomes analogous to that of $g(z)$ in $\S 4$.

The case of $\lambda(z)=z+m$ leads to an $f_{i}(z)$ with a rational addition theorem. Thus far, no attempt seems to have been made to determine all such functions. Given a function with a rational multiplication theorem, we can obtain from it a function with an addition theorem by replacing $z$ by $e^{z}$, or, more generally, by $e^{z} \pi(z)$, where $\pi(z)$ is a periodic function with a suitable period.

\footnotetext{
* Loc. cit.
} 
But it is far from certain that all functions with rational addition theorems can be found in this way.

Given any $f_{i}(z)$ such that $f_{i}[\lambda(z)]$ is rational in $f_{i}(z)$, any $f(z)$ which is rational in $f_{i}(z)$ satisfies with $f[\lambda(z)]$ a relation of genus zero. It is the functions $f(z)$ of this type which give Case (b) under genus zero in the statement of results in the introduction.

11. Preliminaries for Case (c). We start now our investigation of the cases in which the sequences (I) and (II) contain no linear functions. We take an $i_{1}$ so great that for every $i \geqq i_{1}, \phi_{i}^{-1}$ has $r$ branches and $h \leqq 4$ critical points, while $\psi_{i}^{-1}$ has $s$ branches and $k \leqq 4$ critical points.

We shall work under the assumption that $i_{1}=0$. That is, $f_{i_{1}}(z)$ will become a new $f_{0}(z)$. At the end of our work we shall replace $f_{0}(z)$ by an arbitrary rational function of $f_{0}(z)$, thus allowing for the functions $\phi_{1}, \cdots$, $\phi_{i_{1}-1}$ which will have been suppressed.

We assume also that $r \geqq s$. This amounts to replacing, if necessary, $f_{0}(z)$ and $f_{0}[\lambda(z)]$ by $f_{0}(z)$ and $f\left[\lambda^{-1}(z)\right]$. When neither $h$ nor $k$ exceeds 3 , we assume further that $h \geqq k$. $^{*}$

12. Case of $h(z)=e^{z}$. We take the case in which $h=k=2$, and in which $r$ and $s$ are not both 2 . In this case, $\phi_{i}^{-1}$ and $\psi_{i}^{-1}$ have, for every $i$, the same two critical points, $a_{i}$ and $b_{i}$, at each of which their branches are permuted in a single cycle. The two values assumed by $\phi_{i}^{-1}$ and $\psi_{i}^{-1}$ at their critical points are $a_{i+1}$ and $b_{i+1}$, the critical points of $\phi_{i+1}^{-1}$ and $\psi_{i+1}^{-1}$.

As we can use any linear function of $f_{i}(z)$ instead of $f_{i}(z)$ with the same effect, we may replace $\phi_{i}$ and $\psi_{i}$ by any linear function of themselves. We therefore assume, without loss of generality, that $a_{i}=0, b_{i}=\infty$ for every $i$.

It follows immediately that $\phi_{i}(z)=c_{i} z^{ \pm r}$, where $c_{i}$ is a constant. Hence

$$
f_{1}(z)=C_{i}\left[f_{i}(z)\right]^{ \pm r i-1} \text {, }
$$

with $C_{i}$ a constant.

We infer that $f_{1}(z)$ is an integral function devoid of zeros. For if $f_{1}(z)$ had a pole or a zero at $z_{1}$, then $f_{i}(z)(i>0)$ would also have a pole or a zero at $z_{1}$. By taking $i$ large, we could, using (5), make the order of the pole or zero of $f_{1}(z)$ appear arbitrarily large.

Thus $f_{1}(z)=e^{\theta(z)}$, where $g(z)$ is an integral function. Then, since

$$
\phi_{0}(z)=c_{0} z^{ \pm r}, \quad \psi_{0}(z)=d_{0} z^{ \pm \bullet},
$$

* $A$, p. 403. The arrangement of the assumptions with respect to $r$ and $s, h$ and $k$, is different in this paper from that in $A$. In $A$ it was assumed that $h \geqq k$. This is not compatible, when $h=4$, with the assumption that $r \geqq s$. It is desirable in this paper to have $r \geqq s$ when one of the numbers $h$ or $k$ is 4 . We shall still be able to take over from $A$ the discussion of the case of four critical points. 
with $c_{0}$ and $d_{0}$ constants, we have

$$
f_{0}(z)=c_{0} e^{ \pm r \theta(z)}, \quad f_{0}[\lambda(z)]=d_{0} e^{ \pm s g(z)} .
$$

But as $f_{0}[\lambda(z)]=c_{0} e^{ \pm r o[\lambda(z)]}$, we must have

$$
g[\lambda(z)]=p g(z)+q,
$$

with $p= \pm s / r$ and $q=( \pm 1 / r) \log \left(c_{0} / d_{0}\right)$.

Thus, when $h=k=2, f(z)^{*}$ is a rational function of $e^{o(z)}$, where $g(z)$ is an integral function satisfying (6).

Any $f(z)$ which is rational in $e^{o(z)}$, where $g(z)$ obeys (6) for a rational $p$, and any $q$, will satisfy with $f[\lambda(z)]$ a relation of genus zero. It can be shown that if $f(z)$ is not a constant, and if neither $p$ nor $1 / p$ is integral, no $\phi_{i}$ or $\psi_{i}$ will be linear. But not always will $h$ equal 2 . For $f(z)=\cos z$, for instance, as will be seen in the following section, $h=3$. This state of affairs is due to the fact that every $f_{i+1}(z)$ is determined to within a linear function when $f_{i}(z)$ is known, so that, if $f(z)=R\left[e^{g(z)}\right]$, with $R(z)$ rational, $R(z)$ influences the formation of the sequences (I) and (II).

13. Case of $h(z)=\cos z$. In all cases for which $h=3$, we denote the critical points of $\phi_{i}^{-1}$ by $a_{i}, b_{i}, c_{i}$, and the orders of the substitutions which the branches of $\phi_{i}^{-1}$ undergo at $a_{i}, b_{i}, c_{i}$, by $\alpha_{i}, \beta_{i}, \gamma_{i}$ respectively.

We consider now the case in which, for every $i, \alpha_{i}=\beta_{i}=2$. It is proved in $A$ that, at $a_{i}$ and $b_{i}$, the branches of $\phi_{i}^{-1}$ and $\psi_{i}^{-1}$ are permuted in pairs, except that there are two places on the Riemann surfaces of $\phi_{i}^{-1}$ and $\psi_{i}^{-1}$ at $a_{i}$ and $b_{i}$ where the functions are uniform. At $c_{i}$, the branches of the two functions are permuted in single cycles. At the places at $a_{i}$ and $b_{i}$ at which they are uniform, $\phi_{i}^{-1}$ and $\psi_{i}^{-1}$ assume the same pair of values, $a_{i+1}$ and $b_{i+1}$, which are critical points of $\phi_{i+1}^{-1}$ with $\alpha_{i+1}=\beta_{i+1}=2$. Also, at $c_{i}, \phi_{i}^{-1}$ and $\psi_{i}^{-1}$ have a common value, $c_{i+1}$, which is the third critical point of $\phi_{i+1}^{-1}$.

It is easy to see that the branches of the inverse of

$$
F_{i}=\phi_{0} \phi_{1} \cdots \phi_{i}
$$

are permuted in a single cycle at $c_{0}$, that is, $F_{i}(z)$ assumes the value $c_{0}$ only for $z=c_{i}$, and assumes it there $r^{i+1}$ times. It follows that $f_{0}(z)$ never assumes the value $c_{0}$. For $f_{0}(z)$ could equal $c_{0}$ only where $f_{i}(z)$ would equal $c_{i}$, and hence would have to assume the value $c_{0}$ an arbitrarily large number of times wherever it assumed it. Similarly no $f_{i}(z)$ ever equals $c_{i}$.

* $f(z)$ is the rational function of $f_{0}(z)$ mentioned in $\$ 11$. 
We use the linear functions at our disposal in selecting the functions

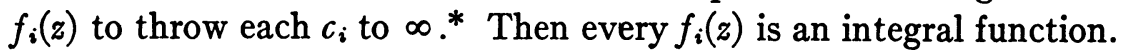

Suppose that wherever $f_{1}(z)$ assumes the values $a_{1}$ and $b_{1}$, it assumes them an even number of times. We use the two constants still at our disposal in choosing $f_{1}(z)$ to arrange so that $a_{1}=1, b_{1}=-1$.

We consider now the function $\arccos z$. In the finite part of the plane it has just two critical points, 1 and -1 , and its branches are permuted in pairs at those points. Also arccos $z$ is never infinite. Hence the expression $\arccos f_{1}(z)$ represents an infinite number of integral functions. Let $j(z)$ be one of these. Then $f_{1}(z)=\cos j(z)$.

We have, a fortiori, $f_{1}(z)=\cos (g(z))^{1 / 2}$, where $g(z)$ is an integral function.

We lay this result aside for the present, and examine the more difficult case in which one of the values $a_{1}$ and $b_{1}$, say $a_{1}$, is assumed an odd number of times at some point $z_{1}$.

There must be a place on the surface of $\phi_{1}^{-1}$ at $a_{1}$ where $\phi_{1}^{-1}$ is uniform and assumes the value $f_{2}\left(z_{1}\right)$. The value $f_{2}\left(z_{1}\right)$ of $\phi_{1}^{-1}$ at this place is the affix of a critical point of $\phi_{2}^{-1}$. As we are free to interchange the letters $a_{i}$ and $b_{i}$ if necessary, we assume that $f_{2}\left(z_{1}\right)=a_{2}$, assigning the letter $b_{2}$ to the second finite critical point of $\phi_{2}^{-1}$. Similarly we let $a_{i}=f_{i}\left(z_{1}\right)$ for every $i$.

We now arrange matters so that $a_{i}=1, b_{i}=-1$ for every $i \geqq 1$.

We have

$$
f_{i+1}(z)=F_{i}^{-1}\left[f_{1}(z)\right],
$$

with $F_{i}=\phi_{1} \cdots \phi_{i}$. The values of $f_{i+1}(z)$ in the neighborhood of $z_{1}$ come from that branch of $F_{i}^{-1}$ which is uniform at $z=1$ and assumes there the value 1 .

We shall study that branch of $F_{i}^{-1}$. Let $n=r^{i}$ be the degree of $F_{i}(z)$. As $\cos z$ assumes the values 1 and -1 twice wherever it assumes them, there exist $n$ integral functions $F_{i}^{-1}(\cos z)$. Let $\alpha(z)$ be that one of these integral functions which equals 1 for $z=0$. As $\alpha(z)$ assumes the values 1 and -1 only through uniform branches of $F_{i}^{-1}$, it assumes those values twice wherever it assumes them. Hence there are an infinite number of integral functions $\arccos \alpha(z)$. It is proved in $A$ that these are all linear. Because arccos $z$ assumes the value 0 at one of its branch points at $z=1$, there are two of the linear functions arccos $\alpha(z)$, negatives of each other, which vanish for $z=0$. Let $t z$ be one of these. Then

$$
\cos z=F_{i}(\cos t z),
$$

so that, because $F_{i}(z)$ is of degree $n$, we have $t= \pm 1 / n$. As we may replace $t$ by $-t$, we assume that $t=1 / n$.

* No difficulty arises when $i=0$; we may also replace $f_{0}(z)$ by a linear function of itself. 
Thus that branch of $F_{i}^{-1}$ which equals 1 for $z=1$ has, for the neighborhood of $z=1$, the representation

$$
F_{i}^{-1}(z)=\cos \frac{\arccos z}{n} .
$$

It is understood here that we use one of those two branches of arccos $z$ which equal 0 for $z=1$.

Consider the integral function

$$
\zeta(z)=\cos z^{1 / 2}
$$

We have, for the neighborhood of $z=1$,

$$
F_{i}^{-1}(z)=\zeta\left[\left(\frac{\arccos z}{n}\right)^{2}\right]
$$

As the two branches of arccos $z$ which vanish for $z=1$ are negatives of each other, the square of either of these branches is uniform at $z=1$. If $z$ describes a path beginning at 1 , and if the two members of (7) are followed along this path, (7) will remain an equation along this path.

We consider now the function

$$
\left[\arccos f_{1}(z)\right]^{2} \text {. }
$$

We shall prove that, if at $z=z_{1}$, where $f_{1}(z)$ assumes the value 1 an odd number of times, we operate on $f_{1}(z)$ with that branch of $(\arccos z)^{2}$ which is uniform at $z=1$, the function (8) obtained is an integral function.

Reject from the plane of $z$ those points other than $z_{1}$ at which $f_{1}(z)= \pm 1$. Let $C$ be the region left after this rejection. Let (8) be continued from $z_{1}$ to any point $z^{\prime}$ in $C$ along a path lying in $C$. While $z$ follows this path, $f_{1}(z)$ describes a path from 1 to $f\left(z^{\prime}\right)$. The function (7) is analytic for all values of $z$ along this path joining 1 to $f_{1}\left(z^{\prime}\right)$.

Now, by (7), we have, since $F_{i}^{-1}\left[f_{1}(z)\right]=f_{i+1}(z)$,

$$
f_{i+1}(z)=1-\frac{\left[\arccos f_{1}(z)\right]^{2}}{2 ! n^{2}}+\frac{\left[\arccos f_{1}(z)\right]^{4}}{4 ! n^{2}}+\cdots
$$

or

$$
n^{2}\left[f_{i+1}(z)-1\right]=-\frac{\left[\arccos f_{1}(z)\right]^{2}}{2 !}+\frac{\left[\arccos f_{1}(z)\right]^{4}}{4 ! n^{2}}+\cdots
$$

Suppose now that for two distinct paths in $C$, leading from $z_{1}$ to $z^{\prime}$, the function $\left[\arccos f_{1}(z)\right]^{2}$ gives two values, $V_{1}$ and $V_{2}$, at $z^{\prime}$. We let $i$ 
increase indefinitely, so that $n=r^{i}$ also approaches $\infty$. All terms other than the first in the second member of (9) will approach 0 , so that, when $i$ is large, the second member will be close to $-V_{1} / 2$ for the first path and close to $-V_{2} / 2$ for the second. But, for every $i$, the first member of (9) is a uniform function. Hence [arccos $\left.f_{1}(z)\right]^{2}$ is uniform (and analytic) in $C$.

When $f_{1}(z)= \pm 1$, $\left[\arccos f_{1}(z)\right]^{2}$ cannot have a singularity if it is uniform, for the branches of $\arccos z$ are all finite for $z= \pm 1$. Thus [arccos $\left.f_{1}(z)\right]^{2}$ is an integral function.

The net result of our discussion is that, whether $f_{1}(z)$ always assumes the values \pm 1 an even number of times, or whether it assumes the value 1 an odd number of times at some point $z_{1}$, we have

$$
f_{1}(z)=\cos (g(z))^{1 / 2}
$$

with $g(z)$ integral. We arrange so that $\phi_{0}(1)=1, \phi_{0}(-1)=-1, \phi_{0}(\infty)=\infty$, and find that

$$
f_{0}(z)=\cos r(g(z))^{1 / 2}, \quad f_{0}[\lambda(z)]=\cos \left[s(g(z))^{1 / 2}+u\right],
$$

where $u=0$ if $\psi_{0}(1)=1$ and $u=\pi$ if $\psi_{0}(1)=-1$. Hence

$$
r(g[\lambda(z)])^{1 / 2}= \pm\left[s(g(z))^{1 / 2}+u\right]+2 t \pi
$$

with $t$ an integer. We have thus

$$
(g[\lambda(z)])^{1 / 2}=p(g(z))^{1 / 2}+q
$$

where $p= \pm s / r$ and where $q=n \pi / r$ with $n$ integral.

An integral $g(z)$ which satisfies (10) with $p$ and $q$ as just described $(r$, $s$, and $n$ are arbitrary) will give an $f_{0}(z)=\cos (g(z))^{1 / 2}$ which satisfies with $f_{0}[\lambda(z)]$ a relation of genus zero. Any $f(z)$ which is rational in $f_{0}(z)$ satisfies with $f[\lambda(z)]$ a relation of genus zero. If neither $p$ nor $1 / p$ is an integer, no $\phi_{i}$ or $\psi_{i}$ will be linear.

If $q$ is chosen different from zero, then $(g(z))^{1 / 2}$ will be integral. For suppose that $(g(z))^{1 / 2}$ is not uniform. Then it vanishes at its branch points. As $(g[\lambda(z)])^{1 / 2}$ has the same branch points as $g(z)$, and vanishes at those points, we must have $q=0$.

When $g(z)$ is integral, it is described as in $\S 4$. If we take $q=0$, any integral $g(z)$ for which $g[\lambda(z)]=p^{2} g(z)$ can be used, whether its square root is integral or not.

14. Case of $h(z)=\varnothing(z)$. We undertake now the investigation of the following two cases:

( $\alpha$ ) Either $h$ or $k$ equals 4 . (It is assumed that $r \geqq s$.)

(及) $h=k=2$. 
When $h=4$ or $k=4$, the branches of $\phi_{i}^{-1}$ and $\psi_{i}^{-1}$ are permuted in pairs at four certain points, except that there are four places on the surface of each function, at those points, where the functions are uniform, the values of $\phi_{i}^{-1}$ and $\psi_{i}^{-1}$ at these places being the critical points of $\phi_{i+1}^{-1}$ and $\psi_{i+1}^{-1}$.

When $h=k=2$, we have $r=s=2$, and the critical points of $\phi_{i}^{-1}$ are two points $a_{i}$ and $b_{i}$, while those of $\psi_{i}^{-1}$ are $a_{i}$ and a third point $c_{i} \neq b_{i}$. The values of $\phi_{i}^{-1}$ at $c_{i}$ are $a_{i+1}$ and $c_{i+1}$ and the values of $\psi_{i}^{-1}$ at $b_{i}$ are $a_{i+1}$ and $b_{i+1}$. There is a point $d_{i}$ at which one value of $\phi_{i}$ is $b_{i+1}$ and at which one value of $\psi_{i}^{-1}$ is $c_{i+1}$. The second values of $\phi_{i}^{-1}$ and $\psi_{i}^{-1}$ at $d_{i}$ are both $d_{i+1}$.

When $h$ or $k$ equals 4 , we represent the critical points of $\phi_{i}^{-1}$ and $\psi_{i}^{-1}$ by $a_{i}, b_{i}, c_{i}$, and $d_{i}$. This will permit us to treat cases $(\alpha)$ and $(\beta)$ together.

Suppose that $f_{1}(z)$ assumes the values $a_{1}$ etc. an even number of times wherever it assumes them. Replacing $f_{1}(z)$ by a linear function of itself if necessary, we suppose that $d_{1}=\infty$, and that $a_{1}+b_{1}+c_{1}=0$. We now construct $\varphi(z)$ with $\varphi\left(\omega_{1}\right)=a_{1}, \wp\left(\omega_{2}\right)=b_{1}, \wp\left(\omega_{3}\right)=c_{1}$. We find that

$$
f_{1}(z)=\varnothing[j(z)]
$$

with $j(z)$ an integral function.* Certainly, then,

$$
f_{1}(z)=\&\left([g(z)]^{1 / 2}\right),
$$

where $g(z)$ is an integral function.

We now take the case in which $f_{1}(z)$ assumes one of the values $a_{1}$ etc. an odd number of times at some point $z_{1}$. If $h=2$, we must have $f_{1}\left(z_{1}\right)=d_{1}$, for the relation

$$
f_{1}[\lambda(z)]=\phi_{1}\left(f_{2}[\lambda(z)]\right)=\psi_{1}\left[f_{2}(z)\right]
$$

shows that $f(z)$ assumes the values $a_{1}, b_{1}, c_{1}$ an even number of times whereever it assumes them. In general, we have $f_{i}\left(z_{1}\right)=d_{i}$.

For $h=4$ or $k=4$, no special assignment of the letters $a_{i}$ etc. to the critical points has been made. We do this now.

We let $d_{1}=f_{1}\left(z_{1}\right)$, and assign the letters $a_{1}, b_{1}, c_{1}$ in any way at all to the other critical points. Then there must be a place at $d_{1}$ at which $\phi_{1}^{-1}$ is uniform and assumes the value $f_{2}\left(z_{1}\right)$. This value of $\phi_{1}^{-1}$ is one of the critical points of $\phi_{2}^{-1}$ or $\psi_{2}^{-1}$. Denoting this critical point by $d_{2}$, we assign the letters $a_{2}, b_{2}, c_{2}$ arbitrarily. We repeat this procedure indefinitely, putting $d_{i}=f_{i}\left(z_{1}\right)$ for every $i$.

Resuming the simultaneous treatment of Cases $(\alpha)$ and $(\beta)$, we suppose that, for every $i \geqq 1, d_{1}=\infty$ and $a_{i}+b_{i}+c_{i}=0$. This means, because $\phi_{i}(z)$

$* A$, p. 425. 
assumes the value $d_{i}$ once at $d_{i+1}$, that each $\phi_{i}(z)$ has a simple pole at $\infty$. We may (and shall) suppose further that the derivative of $\phi_{i}(z)$ equals unity for $z=\infty$. This uses up, for $i>1$, the third constant in the linear transformation at our disposal in choosing $f_{i}(z)$.

We construct, for each $i \geqq 1$, the function $\varphi(z)$ with such periods that its derivative vanishes when $\varnothing(z)$ is $a_{i}, b_{i}$ or $c_{i}$. We represent this function by $\ell_{i}(z)$.

We shall prove that $\wp_{i}(z)=\phi_{i}\left[\varphi_{i+1}(z)\right]$. If we operate on $\varphi_{i}(z)$ with $\phi_{i}^{-1}$, we get $r$ elliptic functions, which assume the values $\infty, a_{i+1}, b_{i+1}$, $c_{i+1}$ twice wherever they assume them. Because $\phi_{i}^{-1}(z)$ has a branch with a simple pole and with derivative unity at $\infty$, one of these elliptic functions has a double pole for $z=0$ with $1 / z^{2}$ for the principal part in its development. Call it $\beta(z)$. Let $\wp_{i+1}^{-1}(z)$ represent the inverse of $\wp_{i+1}(z)$. Then there are an infinitude of linear integral functions $\wp_{i+1}^{-1}[\beta(z)]$. $^{*}$

But $\wp_{i+1}^{-1}$ has one branch point at $\infty$ where its development begins with $z^{-1 / 2}$. One of the two corresponding developments of ${\vartheta_{i+1}}_{i+1}^{-1}[\beta(z)]$ for $z=0$ will start with $z$, and will therefore actually be $z$. This proves that $\varphi_{i}(z)=\phi_{i}\left[\varphi_{i+1}(z)\right]$.

Our next step will be to prove that, as $i$ increases, the periods of $\varphi_{i}(z)$ increase without limit; that is, that given any number $G$, we can find an integer $j$ such that, for every $i>j$, the periods of $\varphi_{i}(z)$ are all at least equal in modulus to $G$.

Let a $G$ be taken. For $i$ sufficiently large, $\wp_{i}(z)$ cannot have two periods with a complex ratio whose moduli are both less than $G$. For in a parallogram based on such periods, $\wp_{i}(z)$ would assume all values at least twice, and $\phi_{1}(z)$, which is a rational function of degree $r^{i-1}$ in $\varphi_{i}(z)$, would assume every value at least $2 r^{i-1}$ times. If $i$ is large, this produces an absurdity.

Every period of $\wp_{i+1}(z)$ is also a period of $\wp_{i}(z)$. Hence, if there is a $\phi_{i}(z)$ with $i$ arbitrarily large, and with a period smaller than $G$ in modulus, there must exist an $\omega$ with $|\omega|<G$ which is a period of $\varphi_{i}(z)$ for every $i$.

Suppose that this is so. Of the numbers $\omega$ which are periods of every $\varphi_{i}(z)$, we select one, $\bar{\omega}$, of smallest modulus. $\dagger$ It is easy to show that

$$
\psi_{i}\left[\wp_{i+1}(z)\right]=\wp_{i}\left[\epsilon_{i} z+u_{i}\right]
$$

where $\epsilon_{i}$ and $u_{i}$ are constants, $2 u_{i}$ being a period of $\ell_{i}(z)$. Because a primitive period parallelogram of $\delta_{i+1}(z)$ has $r$ times the area of a parallelogram for

$* A$, p. 425.

$\dagger$ There will be two such $\bar{\omega}$ 's, negatives of each other. 
$\boldsymbol{\gamma}_{i}(z)$ and $s$ times the area of one for $\wp_{i}\left(\epsilon_{i} z+u_{i}\right)$, we must have $\left|\epsilon_{i}\right|^{2}=s / r$. Hence, as $r \geqq s$, we have $\left|\epsilon_{i}\right| \leqq 1$.

For $i$ large, $\bar{\omega}$ is a smallest period of $\ell_{i}(z)$. Then $\bar{\omega} / \epsilon_{i}$ is a smallest period of $\gamma_{i}\left(\epsilon_{i} z+u_{i}\right)$. As $\bar{\omega}$ is a period of $\varnothing_{i}\left(\epsilon_{i} z+u_{i}\right)$ (it is one of $\wp_{i+1}(z)$ ), we must have $\left|\epsilon_{i}\right| \geqq 1$. Hence $\left|\epsilon_{i}\right|=1$.

Thus, unless $\epsilon_{i}= \pm 1, \varnothing_{i}\left(\epsilon_{i} z+u_{i}\right)$ will have two periods of complex ratio, $\bar{\omega}$ and $\epsilon_{i} \bar{\omega}$, of moduli less than $G$. But if $\epsilon_{i}= \pm 1, \wp_{i}\left(\epsilon_{i} z+u_{i}\right)$ will be a linear function of $\gamma_{i}(z)$, because $u_{i}$ is a half-period, so that $\phi_{i}$ will be a linear function of $\psi_{i}$. This contradicts the fact that $\phi_{i}$ and $\psi_{i}$ are not linear and have no common forefactor. The proof that the periods of $\varphi_{i}(z)$ become infinite with $i$ is thus completed.

Putting $F_{i}=\phi_{1} \cdots \phi_{i}$, we have

$$
f_{i+1}(z)=F_{i}^{-1}\left[f_{1}(z)\right]
$$

For the neighborhood of $z_{1}$, we must use, in (11), that branch of $F_{i}^{-1}$ which equals $\infty$ at $\infty$. We proceed to obtain an expression for that branch.

As $\varnothing_{1}(z)=F_{i}\left[\varphi_{i}(z)\right]$, we have

$$
F_{i}^{-1}(z)=\varphi_{i}\left[\varphi_{1}^{-1}(z)\right] .
$$

To get that branch of $F_{i}^{-1}$ which is infinite at $\infty$, we must use, for $z=0$, one of those two branches of $\varphi_{1}^{-1}(z)$ which vanish at $\infty$.

Let $\zeta_{i}(z)=\wp_{i}(z)^{1 / 2}$. We have

$$
F_{i}^{-1}(z)=\zeta_{i}\left(\left[\varphi_{1}^{-1}(z)\right]^{2}\right) \text {. }
$$

We use here that branch of $\left[{\varphi_{i}^{-1}}^{-1}(z)\right]^{2}$ which is uniform, and equal to zero, at $\infty$. Then, by (11) and (12), we have

$$
f_{i+1}(z)=\zeta_{i}\left(\left[\wp_{1}^{-1}\left\{f_{1}(z)\right\}\right]^{2}\right) \text {. }
$$

For the neighborhood of $z=0$, we have

$$
\zeta_{i}(z)=\frac{1}{z}+l_{1} z+l_{2} z^{2}+\cdots .
$$

Since the periods of $\wp_{i}(z)$ become infinite with $i, \wp_{i}(z)$ degenerates towards $1 / z^{2}$ as $i$ increases. Hence the radius of convergence of the development in (14) increases indefinitely with $i$, while each coefficient $l_{n}$ approaches zero.

We shall now prove that $\left(\wp_{1}^{-1}\left[f_{1}(z)\right]\right)^{2}$ is an integral function. We take any finite region about $z_{1}$, reject from it all points other than $z_{1}$ for which $f_{1}(z)$ equals $\infty, a_{1}, b_{1}$, or $c_{1}$, and let $z$ describe two different paths from $z_{1}$ to any point $z^{\prime}$ in this region. Then, because $\varphi_{1}^{-1}(z)$ is never infinite, 
$\left(\wp_{1}^{-1}\left[f_{1}(z)\right]\right)^{2}$ will describe two bounded paths, which start from 0 and which terminate at two points $V_{1}$ and $V_{2}$.

From (13) and (14), we find

$$
f_{i+1}(z)=\frac{1}{\left(\wp_{1}^{-1}\left[f_{1}(z)\right]\right)^{2}}+l_{1}\left(\wp_{1}^{-1}\left[f_{1}(z)\right]\right)^{2}+\cdots .
$$

For $i$ large enough, the paths leading from 0 to $V_{1}$ and $V_{2}$ will both lie within the circle of convergence of (14). As the coefficients $l_{n}$ approach 0 with $1 / i$, and as the first member of (15) is uniform, $V_{1}$ and $V_{2}$ cannot be distinct. This means that $f_{1}(z)$ is of the form $\varphi_{1}\left[(g(z))^{1 / 2}\right]$ where $g(z)$ is an integral function.

At this point we bring together the case in which $f_{1}(z)$ always assumes the values $a_{1}$ etc. an even number of times with that in which it assumes one of the values somewhere an odd number of times. We put $d_{0}$ equal to $\phi_{0}\left(d_{1}\right),{ }^{*}$ and send $d_{0}$ to $\infty$. Also we make $a_{0}+b_{0}+c_{0}$ equal 0 . Finally, we make $\phi_{0}{ }^{\prime}(\infty)$ unity. Taking a $\varphi(z)$ whose multiple values are $a_{0}$ etc., we see that

$$
f_{0}(z)=\varnothing\left[(g(z))^{1 / 2}\right], \quad f_{0}[\lambda(z)]=\varnothing\left[\epsilon(g(z))^{1 / 2}+u\right]
$$

where $2 u$ is a period of $\varphi(z)$, and $\epsilon$ a constant.

Then

$$
(g[\lambda(z)])^{1 / 2}= \pm \epsilon(g(z))^{1 / 2}+v
$$

where $2 v$ is a period of $\varphi(z)$.

The constant $\epsilon$ must be such that $\varnothing(z)$ and $\varnothing(\epsilon z)$ have a pair of periods in common. It will always be the quotient of two numbers $\epsilon_{1}$ and $\epsilon_{2}$ such

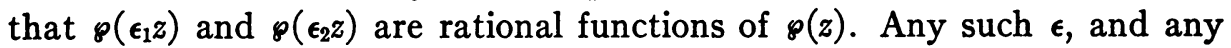
half-period $v$ can be used for the determination of a $g(z)$. If $v \neq 0, g(z)$ must be integral. If $v=0$, we can use any $g(z)$ for which $g[\lambda(z)]=\epsilon^{2} g(z)$, whether $(g(z))^{1 / 2}$ is integral or not.

Any $f(z)$ which is rational in $g(g(z))^{1 / 2}$ satisfies with $f[\lambda(z)]$ an equation of genus zero.

15. The lemniscatic functions. We take the case of $h=3, \alpha_{i}=2$, $\beta_{i}=\gamma_{i}=4$.

Suppose first that wherever $f_{1}(z)$ assumes the value $a_{1}$ it assumes it an even number of times, and that where it assumes the values $b_{1}$ and $c_{1}$ it assumes them a number of times which is divisible by 4 .

* When $h=k=2$, this relation already holds. 
We construct the function $\varnothing(z \mid \omega, i \omega)$, where $\omega$ is any number distinct from zero. For this function, $e_{1}=-e_{3}$. We arrange so that $a_{1}=e_{1}^{2}, b_{1}=0$, $c_{1}=\infty$, and prove that

$$
f_{1}(z)=8^{2}[j(z)]
$$

where $j(z)$ is an integral function. Certainly

$$
f_{1}(z)=\wp^{2}\left[(g(z))^{1 / 4}\right],
$$

where $g(z)$ is integral.

Suppose now that, at $z_{1}, f_{1}(z)$ assumes one of the values $b_{1}, c_{1}$ a number of times which is not divisible by 4 . Let the designation $c_{1}$ be assigned to $f_{1}\left(z_{1}\right)$.

The value $f_{2}\left(z_{1}\right)$ must be assumed by $\phi_{1}^{-1}$ at $c_{1}$ through a uniform branch. This is evident if the value $c_{1}$ is assumed by $f_{1}(z)$ at $z_{1}$ an odd number of times. If $c_{1}$ is assumed at $z_{1}$ an even number of times (not divisible by 4), it might be asked whether $\phi_{1}^{-1}$ does not assume the value $f_{2}\left(z_{1}\right)$ at a simple branch point at $c_{1}$. Such a simple branch point may exist if $r$ is even.* But then $f_{2}\left(z_{1}\right)$ would equal $a_{2} . \dagger$ Also $\phi_{2}^{-1}$ would have no uniform branches at $a_{2}$, and the value $a_{2}$ would be assumed an odd number of times by $f_{2}(z)$ at $z_{1}$. Thus $f_{3}(z)$ could not be uniform at $z_{1}$.

The value $f_{2}\left(z_{1}\right)$, assumed by $\phi_{1}^{-1}$ at a uniform branch at $c_{1}$, is a critical point of $\phi_{2}^{-1}$ at which the branches of $\phi_{2}^{-1}$ undergo a substitution of order 4. We put $f_{2}\left(z_{1}\right)=c_{2}$, whereupon the use of the letter $b_{2}$ is determined. In general, we put $f_{i}\left(z_{1}\right)=c_{i}$.

Taking the function $\varphi(z \mid \omega, i \omega)$, and letting

$$
a_{i}=e_{1}^{2}, \quad b_{i}=0, \quad c_{i}=\infty
$$

for every $i \geqq 1$, it can be proved, using the method of the preceding sections, that

$$
f_{1}(z)=\wp^{2}\left[(g(z))^{1 / 4}\right]
$$

where $g(z)$ is integral. The work is easier than that for the case of four critical points, because we meet in the present case, as in $\$ 13$, multiplication theorems rather than period transformations. No discussion similar to that, in the preceding section, of the size of the periods of $\vartheta_{i}(z)$, is necessary.

\footnotetext{
* $A$, p. 428.

$\dagger A$, p. 428 , line 27 .
} 
In both cases treated above,-in the case in which the value $c_{1}$ is always assumed a number of times divisible by 4 , and in that in which it is not,-we find, putting

$$
\begin{gathered}
a_{0}=e_{1}^{2}, \quad b_{0}=0, \quad c_{0}=\phi_{0}\left(c_{1}\right)=\infty, \\
f_{0}(z)=\wp^{2}\left[\epsilon_{1}(g(z))^{1 / 4}\right], \quad f_{0}[\lambda(z)]=\phi^{2}\left[\epsilon_{2}(g(z))^{1 / 4}+u\right] .
\end{gathered}
$$

Here $\epsilon_{1}$ and $\epsilon_{2}$ are constants such that $\wp^{2}\left(\epsilon_{1} z\right)$ and $\ell^{2}\left(\epsilon_{2} z\right)$ are rational in $q^{2}(z)$, and $u$ is either 0 or $(1+i) \omega$. As to $g(z)$, we must have

$$
\epsilon_{1}(g[\lambda(z)])^{1 / 4}=i^{p} \epsilon_{2}(g(z))^{1 / 4}+v
$$

where $p$ is either $0,1,2$ or 3 , and where $v$ is a constant easy to specify. If $v \neq 0,(g(z))^{1 / 4}$ is integral.

We consider finally the case in which the value $a_{1}$ is assumed by $f_{1}(z)$ at some point $z_{1}$ an odd number of times. We prove as above that

$$
f_{0}(z)=\wp^{2}\left[\epsilon_{1}(g(z))^{1 / 2}+\omega\right], \quad f[\lambda(z)]=\wp^{2}\left[\epsilon_{2}(g(z))^{1 / 2}+u\right]
$$

where $u$ is either $\omega$ or $i \omega$.

16. The equianharmonic functions. In the case of $h=3, \alpha_{i}=\beta_{i}=\gamma_{i}=3$, we construct $\ell(z)$ with $e^{2 \pi i / 3}$ for the ratio of its periods. We prove, assigning appropriate values to $a_{0}, b_{0}, c_{0}$, that

$$
f_{0}(z)=\wp^{\prime}\left[\epsilon_{1}(g(z))^{1 / 3}\right], \quad f_{0}[\lambda(z)]=\wp^{\prime}\left[\epsilon_{2}(g(z))^{1 / 3}+u\right] .
$$

Here $\epsilon_{1}$ and $\epsilon_{2}$ are such that $\wp^{\prime}\left(\epsilon_{1} z\right)$ and $\ell^{\prime}\left(\epsilon_{2} z\right)$ are rational in $\wp^{\prime}(z)$, and $g(z)$ is integral. The constant $u$ is either 0 or a point at which $g^{\prime \prime}(z)=0$. We omit the discussion of $g(z)$.

In the case of $\alpha=2, \beta=3, \gamma=3$ or 6 , we take the function $q(z)$ just constructed and show that

$$
f_{0}(z)=\wp^{3}\left[\epsilon_{1}(g(z))^{1 / n}+u\right], f_{0}[\lambda(z)]=\wp^{3}\left[\epsilon_{2}(g(z))^{1 / n}+u\right],
$$

where $n=6,3$ or 2 . If $n=6, u$ is 0 . If $n=3, u$ is such that $\varphi(u)=0$, while if $n=2,8^{3}(u)=e_{1}^{3}$.

In this section, and in the preceding one, any $f(z)$ which is rational in $f_{0}(z)$ satisfies with $f[\lambda(z)]$ a relation of genus zero.

\section{Columbia University,} New YoRK, N. Y. 